\section{diagenơ̈̈e}

Innovating Epigenetic Solutions

\title{
DNA fragmentation and quality control analysis using Diagenode shearing systems and Fragment Analyzer ${ }^{\mathrm{TM}}$
}

\author{
Optimal data generation using NGS platforms relies on a few sample-preparation prerequisites, namely, precise \\ DNA fragmentation, size-range analysis, and smear quantification. The Diagenode One, Bioruptor ${ }^{\circledast}$, Megaruptor $^{\circledast}$, \\ and Fragment Analyzer ${ }^{\mathrm{TM}}$ by Advanced Analytical ensure that these first critical steps generate quality results.
}

NGS technologies have revolutionized genomics research ${ }^{1}$. Optimal DNA shearing, sizing, and quantification during library construction are critical to generate high-quality sequencing data. DNA must be efficiently sheared to an appropriate and consistent fragment size (depending on the sequencing platform) and accurately analyzed to measure distributions and molarity concentrations. The combined use of Diagenode's shearing instruments with Advanced Analytical's Fragment Analyzer ${ }^{\mathrm{TM}}$ can optimize small-fragment (100 bp-1 kb) and long-fragment (2-75 kb) NGS library preparation workflows (Fig. 1).

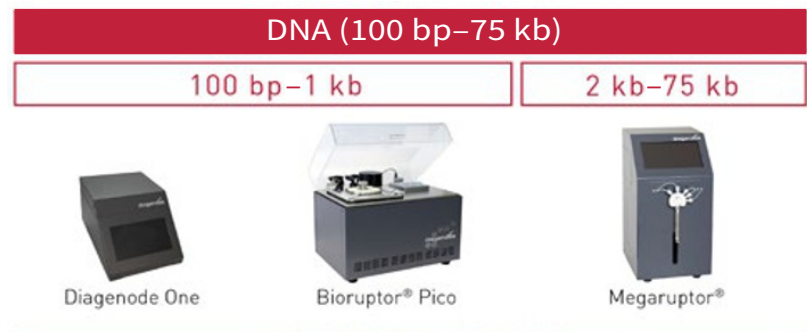

DNA (75 bp-50 kb)

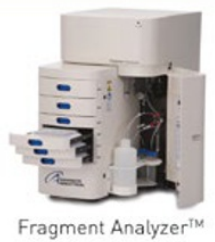

Figure 1 | DNA template generation: breaking and sizing samples. Top: the Diagenode One, the Bioruptor ${ }^{\oplus}$ Pico and the Megaruptor ${ }^{\oplus}$ provide superior sample yields, fragment distribution, and consistency. Bottom: the Fragment Analyzer $^{\mathrm{rm}}$ generates accurate sizing and molarity data for DNA up to $50 \mathrm{~kb}$, with an optimized workflow essential for NGS.

Wassim Lakhal ${ }^{1}$, Irina Panteleeva ${ }^{1}$, Sharon Squazzo ${ }^{2}$, Rini Saxena ${ }^{2}$, Jerome Kroonen ${ }^{1}$, Steve Siembieda ${ }^{3}$, Markus Tilmes $^{3}$ \& Jonathan Hagopian 3

${ }^{1}$ Diagenode s.a., Liège, Belgium. ${ }^{2}$ Diagenode Inc., Denville, New Jersey, USA. ${ }^{3}$ Advanced Analytical Technologies, Inc., Ankeny, lowa, USA. Correspondence should be addressed to J.K. (jerome.kroonen@diagenode.com) or J.H. (info@aati-us.com).
Precisely shear, size, and quantify DNA down to $100 \mathrm{bp}$ for short-read sequencing

DNA can be sheared into small fragments (down to $100 \mathrm{bp}$ ) through cavitation via sonication. The Bioruptor ${ }^{\circledR}$ Pico and Diagenode One both use this technology, making these instruments optimal for the homogeneous and reproducible shearing of DNA samples. As cavitation generates heat, both devices are equipped with an appropriate cooling system, preventing sample degradation and ensuring high sample recovery. The uniform ultrasounds facilitate unbiased shearing and high yields of double-stranded DNA. To confirm shearing results, high-resolution analysis of DNA size, quantity, and quality is recommended. The Fragment Analyzer ${ }^{\mathrm{TM}}$ automates analysis of the quality and quantity of library preparations and eliminates handson steps. The Fragment AnalyzerTM simplifies library QC owing to its capability to hold up to 288 samples at one time and its ability to separate 12,48 , or 96 samples in parallel, making it the premier parallel capillary electrophoresis instrument. With a high-quality syringe pump to deliver reagents automatically, a powerful LED to excite the intercalating dye, and a CCD (charge-coupled device) camera that continuously monitors for fluorescent events, the Fragment Analyzer ${ }^{\top \mathrm{M}}$ is unmatched in terms of sensitivity and resolution. With over 20 reagent kits available, all types of DNA and RNA samples can be effectively separated for any application or throughput requirement.

The Diagenode One is the smallest and lightest shearing instrument available. The microfluidic chips used with the instrument provide optimal small-volume (20 and $50 \mu \mathrm{l}$ ) shearing. Independent DNA samples are prepared with the Diagenode One and subsequently assessed with the Fragment Analyzer ${ }^{\mathrm{TM}}$ (Fig. 2a).

The Bioruptor ${ }^{\circledR}$ Pico is the latest innovation in DNA shearing, capable of effectively sonicating micro-volumes of $5 \mu \mathrm{l}$ to larger volumes of $2 \mathrm{ml}$. The Bioruptor Pico provides accurate and consistent DNA fragmentation of up to 12 samples simultaneously, and it is capable of shearing samples down to $200 \mathrm{bp}$ in less than $15 \mathrm{~min}$. The system is easily tunable, as illustrated in Figure $\mathbf{2 b}$, which demonstrates the ability of the Bioruptor Pico to shear fragments down to $200 \mathrm{bp}, 350 \mathrm{bp}$, or $1 \mathrm{~kb}$. Integrating the Bioruptor Pico and the Fragment Analyzer ${ }^{\mathrm{TM}}$ in the NGS laboratory workflow dramatically simplifies and improves NGS sample preparation. 


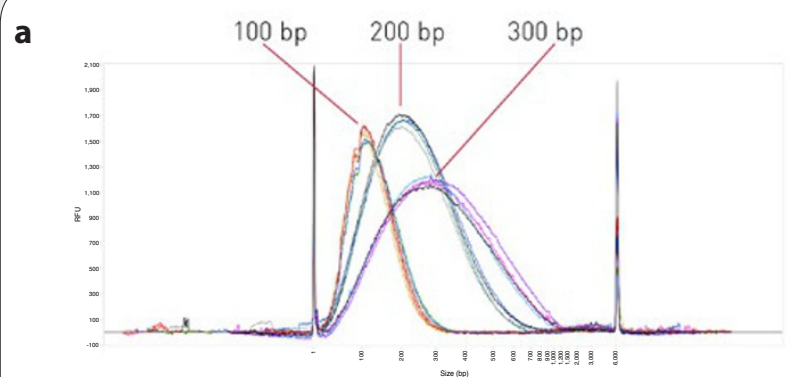

200 bp (13 cycles 30" / 30")

b

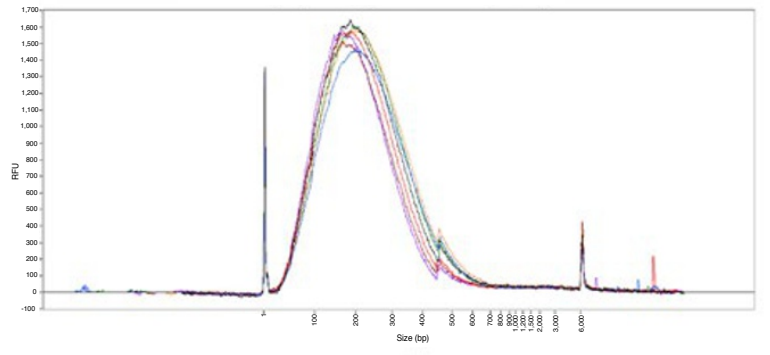

350 bp (5 cycles 30" / 90")

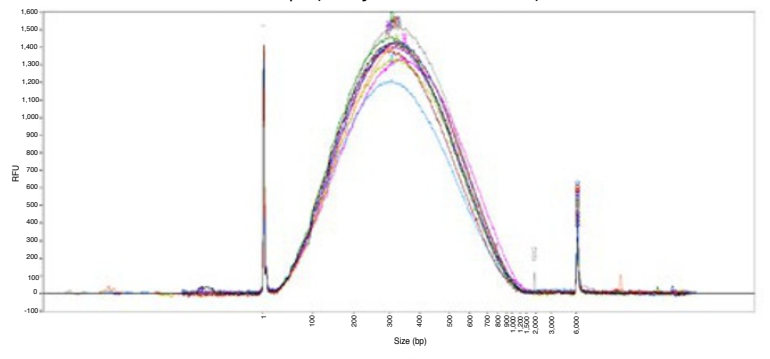

Figure $\mathbf{2}$ | Unbiased and accurate DNA shearing and sizing for NGS. (a,b) Human genomic DNA (10-20 ng/ $\mu \mathrm{l}$, 50 or $100 \mu$ per sample) was sonicated using (a) the Diagenode One sonication device or (b) Bioruptor Pico highthroughput. Small fragments were prepared for NGS (100 bp-1 kb) using different sonication conditions. Sheared DNA was analyzed using the Fragment Analyzer ${ }^{\mathrm{TM}}$ and the DNF-474 High Sensitivity NGS Fragment Analysis Kit (1-6,000 bp) from Advanced Analytical. RFU, relative fluorescence units.

\section{Accurately shear, size, and quantify DNA up to $50 \mathrm{~kb}$ for long-read sequencing}

Technological advances in long-read DNA sequencing have enabled researchers to investigate increasingly intricate questions about the arrangement of complex genomic elements. In order to take full advantage of the technological advances in long-read DNA sequencing, scientists need supporting technologies able to effectively create and analyze large fragment libraries. The construction of a long-read sequencing library depends on a number of critical parameters including the quality and quantity analysis of the input genomic DNA, shearing of genomic DNA, and control of the fragmentsize distribution before final size selection and analysis. A number of commercial solutions currently provide limited options, able only to prepare libraries under $20 \mathrm{~kb}$ in size. Now, through the combined use of the Megaruptor ${ }^{\circledR}$ and Fragment Analyzer ${ }^{\text {TM }}$, large fragment libraries up to $50 \mathrm{~kb}$ in size can be accurately prepared and quickly resolved. The Megaruptor uses unique hydropore disposables able to attain a precise fragment distribution within a twofold to threefold size range. This tight distribution can be analyzed in just $1 \mathrm{~h}$ with the Fragment Analyzer ${ }^{\mathrm{TM}}$ (Fig. 3).

The Megaruptor pumps the sample DNA through an array of uniform hydropores; the resulting turbulent flow stretches and breaks the DNA strands. Two types of hydropores with specific pore sizes are available. Short hydropores are for fragments from 2 to $9 \mathrm{~kb}$ (Fig. 3), whereas long hydropores shear DNA strands from $10 \mathrm{~kb}$ to $75 \mathrm{~kb}$ (Fig. 3). This novel design eliminates clogging and cross-contamination, as the shearing is independent of DNA source, DNA concentration, and salt content. The desired size is selected through accompanying software that automatically sets the solution flow rate to get high-quality ultra-long DNA molecules. Post-fragmentation sizing with the Fragment AnalyzerTM eliminates long wait times for quality-control analysis. The Fragment Analyzer ${ }^{\mathrm{TM}}$ can accurately separate and size large fragment smears in $1 \mathrm{~h}$, eliminating the need for overnight pulsedfield gel electrophoresis (PFGE). To date, multiple overnight PFGE steps can be eliminated with the Fragment Analyzer ${ }^{\mathrm{TM}}$, dramatically reducing the time required for library preparation. Additionally, analysis of large fragment smears requires only 2 ng of library DNA—a $>25$-fold reduction compared with agarose-based PFGE_conserving sample for sequencing and ensuring minimal sample loss.
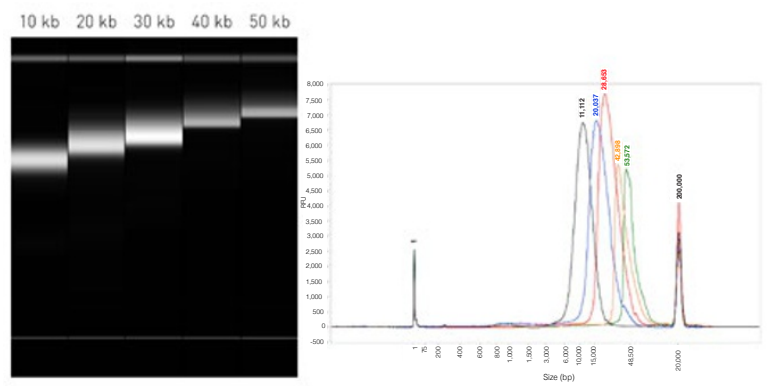

Figure 3 | Preparation of long DNA molecules for real-time sequencing (10-50 kb). Human genomic DNA samples ( $25 \mathrm{ng} / \mu \mathrm{l}, 200 \mu \mathrm{l}$ per sample) were sheared mechanically with the Megaruptor ${ }^{\circledast}$. Fragment Analyzer ${ }^{\mathrm{TM}}$ profiles of DNA sheared using long hydropores and different software settings of 10, 20, 30, 40, and $50 \mathrm{~kb}$, analyzed with the DNF-464 High Sensitivity Large Fragment 50 kb Analysis Kit. RFU, relative fluorescence units.

\section{Conclusion}

Unbiased DNA fragmentation with the Diagenode One, the Bioruptor ${ }^{\circledR}$ Pico, or the Megaruptor ${ }^{\circledR}$ for short- or long-read sequencing combined with sizing on the Fragment Analyzer ${ }^{\text {TM }}$ delivers an efficient workflow solution for preparing and analyzing libraries with even distribution and representation of the genome.

1. Goodwin, S., McPherson, J.D. \& McCombie, W.R. Nat. Rev. Genet. 17, 333-351 (2016).

This article was submitted to Nature Methods by a commercial organization and has not been peer reviewed. Nature Methods takes no responsibility for the accuracy or otherwise of the information provided. 